\title{
ON EXACT BOUNDS FOR THE CLUSTER FREE ENERGY IN THE THREE-DIMENSIONAL LATTICE-GAS MODEL*
}

\author{
R. TORAL and J. MARRO \\ Departamento de Física Teórica, Universidad de Barcelona, Diagonal 647, 08028 Barcelona, Spain
}

Received 9 July 1985

\begin{abstract}
The exact bounds by Lebowitz and Penrose for the cluster distribution of the simple cubic lattice-gas model with attractive interactions lead here to exact upper and lower bounds for the limiting cluster free energy or free energy per particle of a cluster of macroscopic size and, consequently, for the renormalized fugacity relating cluster distribution and partition functions. We also give practical realizations of the bounds for the cluster distribution. Our upper and lower bound expressions are always close enough to each other for $4 J / k_{\mathrm{B}} T>1.5$. The nature of the bounds at higher temperatures is also analysed.
\end{abstract}

\section{Introduction}

A given configuration of the lattice-gas version of the Ising model can be described in terms of clusters defined, for instance, as maximal sets of connected occupied sites. For equilibrium states, the probabilities for the occurrence of these clusters are governed by the corresponding Gibbs distribution. Such a simple picture of the system as a collection of non-overlapping clusters is very convenient in the theory of nucleation ${ }^{1}$ ), e.g. to describe the decay of a metastable state. This is done in practice via the concept of the cluster free energy, $F_{l}$, which is defined as

$$
F_{l}=-k_{\mathrm{B}} T \ln Q_{l} .
$$

Here $k_{\mathrm{B}}$ is the Boltzmann constant, $T$ represents the temperature, and $Q_{l}$ is the partition function for the $l$-particle clusters $\left.{ }^{2}\right)$. The equilibrium cluster distribution, $c_{l}$, that is the number of $l$-particle clusters per lattice site as a function of the "size" $l$ in the equilibrium state, is in principle related to the partition function $Q_{l}$. A semiphenomenological relation of this kind is ${ }^{3}$ )

*'Partially supported by the US-Spanish Cooperative Research Program, Grant CCB-8402025. 


$$
c_{l}=\omega^{l} Q_{l}(1-\rho)^{k_{l}},
$$

where $\rho$ is the system density (number of particles per lattice site), $\omega=\omega(\rho, T)$ is a renormalized fugacity and the parameter $k_{l}$ has a weak dependence on $l$ for very small $l$ (e.g. $k_{1}=3.25, k_{2}=4.5, k_{l}=5, l \geqslant 3{ }^{4}$ ).

Dickman and Schieve ${ }^{5}$ ) proved the existence of the limiting cluster free energy or free energy per particle for a cluster of macroscopic size, i.e. $q \equiv \lim _{l \rightarrow \infty}\left(\ln Q_{l} / l\right)$. The proof in ref. 5 uses ideas from the derivation by Penrose and Lebowitz ${ }^{6}$ ) of exact upper and lower bounds for the cluster free cncrgy. Thesc bounds, however, are often crude in practice and, consequently, this fact limits severely the utility of the resulting values for the limiting free energy $q$. In this paper we obtain upper and lower bounds for $q$ which are useful for temperatures below $0.6 T_{\mathrm{c}}, T_{\mathrm{c}}$ being the corresponding critical temperature. We also deduce some interesting bounds for the renormalized fugacity in eq. (2) and give practical realizations of existing exact bounds for the cluster distribution.

\section{Limiting free energy and renormalized fugacity}

Our results apply to the familiar lattice-gas or Ising model in a simple cubic lattice with $N$ sites. At each site $i=1,2, \ldots, N$ of the lattice there is defined an occupation variable $n_{i}$ with two values, either 1 (particle) or 0 (unoccupied site), and there is an attractive interaction between particles; each configuration $C \equiv\left\{n_{i}\right\}$ has a configurational energy defined as

$$
E\{C\}=-4 J \sum_{i, j}^{\prime} n_{i} n_{j}, \quad J>0,
$$

where the sum runs over all the nearest-neighbor pairs of sites. One may introduce the grand partition function at temperature $T$ and fugacity $z$,

$$
\Xi(z, T)=\sum_{\{C\}} z^{\rho N} \exp \left[-E\{C\} / k_{\mathrm{B}} T\right],
$$

where the sum runs now over all the $2^{N}$ possible configurations. Here $\rho N=\Sigma_{i} n_{i}$ is the number of particles in configuration $C$. The probability for the occurrence at equilibrium of a given configuration $C$ is then given by $P(C)=$ $\Xi^{-1} \exp \left[-E\{c\} / k_{\mathrm{B}} T\right]$.

We shall need the following definitions concerning a given cluster $K$ :

$-\Lambda$ is the set of lattice sites.

$-B(K)$ is the border of $K$, i.e. the set of sites which are nearest neighbors of any site in $K$ and do not belong to $K$. 
$-\bar{K}=K \cup B(K)$.

$-N(K), N(B(K))$ and $N(\bar{K})$ are, respectively, the number of particles in $K$, $B(K)$ and $\bar{K} ; N(\bar{K})=N(K)+N(B(K))$.

$-b(l)=\min _{K \cdot N(K)=l} N(B(K))$, i.e. the minimum value of the number of sites in the border of any $l$-particle cluster.

$-E(K)=-4 J \sum_{i, j \in K}^{\prime} n_{i} n_{j}$ is the energy of the cluster.

$-\theta(K)=z^{N(K)} \exp \left[-E(k) / k_{\mathrm{B}} T\right]$.

$-Q_{l}=\sum_{K: N(K)=l} \exp \left[-E(K) / k_{\mathrm{B}} T\right]=z^{-N(K)} \sum_{K: N(K)=l} \theta(k) ; Q_{l}$ only depends on the temperature and it can be written as a polynomial in $y \equiv$ $e^{\gamma}\left(\gamma \equiv 4 J / k_{\mathbf{B}} T\right): Q_{l}=\sum_{m} Q_{l}(m) y^{x_{m}}$.

- $\Xi(K)$ is the grand partition function for a lattice-gas confined to the sites of $K$.

The bounds we shall obtain for $q$ follow from the ones for $c_{l}$ by Lebowitz and Penrose [2]. These bounds for $c_{l}$ will be analyzed later on; for the moment it suffices to consider their crudest form, i.e.

$$
Q_{l} z^{l}(1-\rho)^{2+5 l} \leqslant c_{l} \leqslant Q_{l} z^{l}(1+z)^{-l-b(l)} .
$$

This expression was the base for eq. (2) ${ }^{3}$ ); it can also be written as

$$
\begin{aligned}
\ln z-\left(\frac{2}{l}+5\right) \ln (1-\rho) & \geqslant \frac{\ln Q_{l}}{l}-\frac{\ln c_{l}}{l} \\
& \geqslant\left(1+\frac{b(l)}{l}\right) \ln (1+z)-\ln z .
\end{aligned}
$$

Let us consider a phase point at "zero field", i.e. on the coexistence curve above or below $T_{\mathrm{c}}$, and denote by $\omega_{0}, \rho_{0}, z_{0}, c_{l}^{0}, \ldots$ the corresponding values of $w, \rho, z, c_{l}, \ldots$, respectively. We then introduce the limit $l \rightarrow x$ in eq. (6) and use the following two lemmas:

Lemma 1. $\lim _{l \rightarrow \infty}\left(\ln c_{l}^{0} / l\right)=0$. This follows from a rigorous result by Kunz and Souillard ${ }^{7}$ ) stating that $\ln c_{l}^{0} \sim l^{2 / 3}$ as $l \rightarrow \infty$.

Lemma 2. $\lim _{l \rightarrow \infty}[b(l) / l]=0$. The proof is as follows: Let $K$ be a cluster, $N(K)=l$, and $L \equiv\left[l^{1 / 3}\right]+1([a]$ is largest integer contained in $a)$.

For some very compact cluster, $\bar{K}$ will be contained in a box of side $L+2$, and $N(\bar{K}) \leqslant(L+2)^{3}$. Then, $N(B(K))=N(\bar{K})-N(K) \leqslant(L+2)^{3}-l<9 l^{2 / 3}$. As $b(l)$ is the minimum value of $N(B(K))$, one has $b(l) \leqslant N(B K))<9 l^{2 / 3}, b(l) / l<$ $9 l^{-1 / 3}$, and the lemma follows. 
By taking the limit $l \rightarrow \infty$ in eq. (6) and using the above two lemmas we finally have

$$
-5 \ln \left(1-\rho_{0}\right) \geqslant q-3 \gamma \geqslant \ln \left(1+\mathrm{e}^{-3 \gamma}\right),
$$

where $z_{0}=\mathrm{e}^{-3 \gamma}$ for a simple cubic lattice. These limits are in fact useful at low temperatures, say $T \leqslant 0.6 T_{c}$; for example, they give $4.573 \geqslant q \geqslant 4.511$ at $\gamma=1.5$ and become even better for higher values of $\gamma$. This agrees with the value $q=4.513$ given by Perini et al. ${ }^{8}$ ). See fig. 1 .

We now need the following lemma:

Lemma 3. ("Soltz criterium" " $)$ ). If $\left(q_{l}\right)$ is a succession in $l$, then $\lim _{l \rightarrow \infty}\left(q_{l} l\right.$ $l)=\lim _{l \rightarrow \infty}\left(q_{l}-q_{l-1}\right)$. The proof is as follows: let $\alpha_{l} \equiv q_{l}-q_{l-1}\left(q_{0} \equiv 0\right)$ and $\alpha \equiv \lim _{l \rightarrow \infty} \alpha_{l}$. This means that for any $\varepsilon>0$, there exists an $N_{0}$ such that if $l>N_{0}$ then $\alpha_{l}=\alpha+\delta_{l}$ with $\left|\delta_{l}\right|<\varepsilon$. Let $N_{1}=\max \left(N_{0}, \varepsilon^{-1}\left|\delta_{1}+\cdots+\delta_{N_{0}}\right|\right)$. Now, if $l>N_{1}$ then $q_{l} / l=\left(\alpha_{1}+\alpha_{2}+\cdots+\alpha_{l}\right) / l=\alpha+\left(\delta_{1}+\cdots+\delta_{N_{0}}\right) / l+\left(\delta_{N_{0}+1}+\right.$ $\left.\cdots+\delta_{l}\right) / l$. The last two terms here are respectively less than $\mid \delta_{1}+\cdots+$ $\delta_{N_{0}}\left|/ N_{1}<\right| \delta_{1}+\cdots+\delta_{N_{0}}\left|/ \varepsilon^{-1}\right| \delta_{1}+\cdots+\delta_{N_{0}} \mid=\varepsilon$ and less than $\varepsilon\left(l-N_{0}^{-1}\right) / l<\varepsilon$, so that it finally follows that $\left|q_{l} / l-\alpha\right|<2 \varepsilon$ and $\lim _{l \rightarrow \infty}\left(q_{l} / l\right)=\alpha$. Q.E.D.

The renormalized fugacity at coexistence, $\omega_{0}$, can be obtained in practice ${ }^{3}$ ) as the radius of convergence of the series

$$
\rho=\sum_{l=1}^{\infty} l \omega^{l} Q_{l}(1-\rho)^{k_{l}} .
$$

That is, $\omega_{0}=\lim _{l \rightarrow \infty}\left(Q_{l} / Q_{l+1}\right)$ and the lemma 3 implies $-\ln \omega_{0}=$ $\lim _{l \rightarrow \infty}\left(\ln Q_{l} / l\right) q$. From eq. (7) we then get

$$
z_{0}\left(1-\rho_{0}\right)^{5} \leqslant \omega_{0} \leqslant z_{0} /\left(1+z_{0}\right) .
$$

This gives $0.0103 \leqslant \omega_{0} \leqslant 0.0110$ at $\gamma=1.5$, thus including the value $\omega_{0}=0.01056$ obtained previously as an empirical fit to eqs. (8) and (2) ${ }^{4}$ ).

\section{Cluster distribution bounds}

Lebowitz and Penrose ${ }^{2}$ ) have established more accurate bounds than those in eq. (5) for the probability $c(K)$ of occurrence of a given cluster $K$. These bounds are based on the inequalities by Fortuin, Kasteleyn and Ginibre $\left.{ }^{9}\right]$ which apply (among others) to systems with attractive two-body interactions. The bounds 
read as follows:

Lower bounds:

$$
\begin{aligned}
& c(K) \geqslant \theta(K) \prod_{i \in \bar{K}}(1-\rho), \\
& c(K) \geqslant \frac{\theta(K)}{\Xi(K)} \prod_{i \in B(K)}(1-\rho) .
\end{aligned}
$$

Upper bounds:

$$
\begin{aligned}
& c(K) \leqslant \frac{\theta(K)}{\Xi(K)} \prod_{i \in B(K)}(1+z)^{-1}, \\
& c(K) \leqslant \theta(K) \prod_{i \in K}(1+z)^{-1}, \\
& c(K) \leqslant \theta(K)\left\{\Xi(K)\left[1+\sum_{i \in \bar{K}} \sum_{j \in \Lambda-\bar{K}}\left(\frac{z}{1+z}\right)^{2}\left(\mathrm{e}^{-\beta V_{i j}}-1\right)\right]\right\} .
\end{aligned}
$$

$V_{i j}$ is the interaction energy between sites $i$ and $j$; it is equal to $-4 J$ when $(i, j)$ are nearest neighbors and 0 otherwise. By realizing that $c_{l}=\Sigma_{K: N(K)=l} c(K)$, a detailed study of all the clusters $K$ up to a size $N(K)=5$ gives the following realizations of eqs. (10) and (11):

From eq. (10a) $\left(y \equiv \mathrm{e}^{\gamma}\right)$ :

$$
\begin{aligned}
c_{1} \geqslant & z(1-\rho)^{7}, \\
c_{2} \geqslant & 3 z^{2} y(1-\rho)^{12} \\
c_{3} \geqslant & 3 z^{3} y^{2}(1-\rho)^{16}(5-\rho), \\
c_{4} \geqslant & z^{4} y^{3}(1-\rho)^{19}\left[83-105 \rho+33 \rho^{2}-3 \rho^{3}+3 y(1-\rho)\right], \\
c_{5} \geqslant & 3 z^{5} y^{4}(1-\rho)^{22}\left[8\left(2-3 \rho+\rho^{2}\right) y+162-346 \rho+278 \rho^{2}\right. \\
& \left.-102 \rho^{3}+17 \rho^{4}-\rho^{5}\right] .
\end{aligned}
$$

From eq. (10b):

$$
\begin{aligned}
& c_{1} \geqslant \frac{z}{1+z}(1-\rho)^{6}, \\
& c_{2} \geqslant \frac{3 z^{2} y(1-\rho)^{10}}{1+2 z+z^{2} y},
\end{aligned}
$$




$$
\begin{aligned}
c_{3} \geqslant & \frac{3 z^{3} y^{2}(1-\rho)^{13}(5-\rho)}{1+3 z+(1+2 y) z^{2}+z^{3} y^{2}}, \\
c_{4} \geqslant & z^{4} y^{3}(1-\rho)^{15}\left\{\frac{3 y(1-\rho)}{1+4 z+2(1+2 y) z^{2}+4 z^{3} y^{2}+z^{4} y^{4}}\right. \\
& +\frac{3\left(21-31 \rho+11 \rho^{2}-\rho^{3}\right)}{1+4 z+3(1+y) z^{2}+2 y(1+y) z^{3}+z^{4} y^{3}} \\
& \left.+\frac{4(5-3 \rho)}{1+4 z+3(1+y) z^{2}+\left(1+3 y^{2}\right) z^{3}+z^{4} y^{3}}\right\} \\
c_{l} \geqslant & -\frac{(1-\rho)^{4 l+2} Q_{l} z^{l}}{1+\left(\begin{array}{c}
l \\
1
\end{array}\right) z+\left(\frac{l}{2}\right) z^{2} y+\cdots+\left(\begin{array}{c}
l \\
m
\end{array}\right) z^{m} y^{x_{m}}+\cdots}
\end{aligned}
$$

From eq. (11a) $\left(p \equiv(1+z)^{-1}\right)$ :

$$
\begin{aligned}
c_{1} \leqslant & z p^{7}, \\
c_{2} \leqslant & \frac{3 z^{2} y p^{10}}{1+2 z+z^{2} y}, \\
c_{3} \leqslant & \frac{3 z^{3} y^{2} p^{13}(p+4)}{1+3 z+(1+2 y) z^{2}+z^{3} y^{2}}, \\
c_{4} \leqslant & z^{4} y^{3}\left\{\frac{3 p^{16}\left(p^{2}+8 p+12\right)}{1+4 z+3(1+y) z^{2}+2 y(1+y) z^{3}+z^{4} y^{3}}\right. \\
& \left.+\frac{4 p^{15}(3 p+2)}{1+4 z+3(1+y) z^{2}+\left(1+3 y^{2}\right) z^{3}+z^{4} y^{3}}\right\} \\
& +\frac{3 z^{4} y^{4} p^{16}}{1+4 z+(1+2 y) 2 z^{2}+4 z^{3} y^{2}+z^{4} y^{4}} .
\end{aligned}
$$

From eq. (11b):

$$
\begin{aligned}
& c_{1} \leqslant z p^{7}, \\
& c_{2} \leqslant 3 z^{3} y p^{12}, \\
& c_{3} \leqslant 3 z^{3} y^{2} p^{16}(p+4), \\
& c_{4} \leqslant 3 z^{4} y^{4} p^{20}+z^{4} y^{3} p^{19}\left[3 p^{3}+24 p^{2}+48 p+8\right], \\
& c_{5} \leqslant 24 z^{5} y^{5} p^{23}(p+1)+3 z^{5} y^{4} p^{22}\left(8+33 p+64 p^{2}+44 p^{3}+12 p^{4}+p^{5}\right) .
\end{aligned}
$$




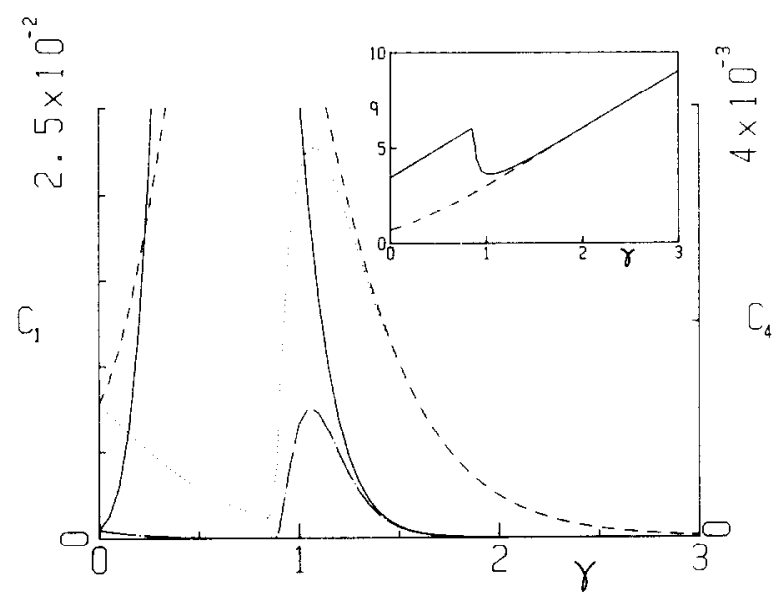

Fig. 1. Behavior with the inverse temperature $\gamma=4 J / k_{\mathrm{B}} T$ of the bounds in this paper: the upper bound for $c_{1}(-\ldots--)$ as given by eq. (16), the lower bound for $c_{1}(\ldots \ldots \ldots)$ as given by eq. (15), the upper bound for $c_{4}\left(-\_\right.$) as given by eq. (14) and the lower bound for $c_{4}\left(-\cdot-{ }_{-}\right)$ as given by eq. (13). The curves for the upper bounds present rather broad peaks below $T_{\mathrm{c}}$ which are not shown in the figure. $T_{\mathrm{c}}$ corresponds to the minimum in the curves for the lower bounds. The inset shows the bounds in eq. (7) for the limiting cluster free energy $q$.

And, from eq. (11c):

$$
\begin{aligned}
c_{1} \leqslant & z\left[1+18(y-1) z^{2} p^{2}\right]^{-1} /\left[1+7 z+(2 y+5) 3 z^{2}+(3 y+4) 5 z^{3}\right. \\
& \left.+(4 y+3) 5 z^{4}+(5 y+2) 3 z^{5}+7 z^{6}+z^{7}\right] \\
c_{2} \leqslant & 3 y z^{2}\left[1+26(y-1) z^{2} p^{2}\right]^{-1}\left[1+12 z+(15 y+51) z^{2}\right]^{-1} .
\end{aligned}
$$

The above expressions are in fact useful realizations of the bounds in eqs. (10) and (11), i.e. they are close enough to each other at low temperatures, say $\gamma \geqslant 1.5$. Fig. 1 depicts the typical behavior with temperature of the above bounds.

\section{References}

1) See, for instance, J. Gunton and M. Droz, Introduction to the Theory of Metastable and Unstable States (Springer, Berlin, 1983).

2) J.L. Lebowitz and O. Penrose, J. Stat. Phys. 16 (1977) 321.

3) M. Kalos, J. Lebowitz, O. Penrose and A. Sur, J. Stat. Phys. 18 (1978) 39.

4) J. Marro and R. Toral, Physica 122A (1983) 563.

5) R. Dickman and W. Schieve, J. Stat. Phys. 36 (1984) 435

6) O. Penrose and J. Lebowitz, in: Fluctuation Phenomena, E.W. Montroll and J.L. Lebowitz, eds. (North-Holland, Amsterdam 1979), p. 293.

7) H. Kunz and B. Souillard, Phys. Rev. Lett. 40 (1978) 133; J. Stat. Phys. 19 (1978) 77.

8) A. Perini, G. Jacucci and G. Martin, Phys. Rev. B 29 (1984) 2689.

9) J. Rey Pastor, Elementos de Análisis Algebraíco (Editorial Martin, Madrid, 1958), p. 362.

10) C. Fortuin, P. Kasteleyn and J. Ginibre, Comm. Math. Phys. 22 (1971) 89. 